(c) 2003 American Chemical Society, Inorg. Chem., Pan ic0300159 Supporting Info Page 1

\title{
Supplementary Materials
}

PRIVILEGED DOCUMENT REVISED REC'D

S-Table 1. Phosphorescence wavelengths and the natural atomic orbital populations of the excitedEP 042003 state and the corresponding ground state for head-to-head $\left[\mathrm{Au}\left(\mathrm{PH}_{2} \mathrm{CH}_{2} \mathrm{SH}\right)\right]_{2}{ }^{2+}(3)$ and head-tohead $\left[\mathrm{Au}\left(\mathrm{PH}_{2} \mathrm{CH}_{2} \mathrm{SH}\right)\right]_{2}{ }^{2+} \cdot(\mathrm{MeCN})_{2}$ (4) under the CIS calculations.

INORG CHEM

\begin{tabular}{|c|c|c|c|c|c|}
\hline \multirow{2}{*}{ Atom } & \multirow{2}{*}{ Orbitals } & \multicolumn{2}{|c|}{3} & \multicolumn{2}{|c|}{4} \\
\hline & & Ground-state & Excited-state & Ground-state & Excited-state \\
\hline \multirow[t]{4}{*}{ Aul } & $6 s$ & 0.774 & 0.753 & 0.665 & 0.687 \\
\hline & $6 \mathrm{p}$ & 0.014 & 0.025 & 0.022 & 0.044 \\
\hline & $5 d$ & 9.829 & 9.790 & 9.819 & 9.637 \\
\hline & $4 f$ & 0.002 & 0.003 & 0.003 & 0.004 \\
\hline \multirow[t]{4}{*}{$\mathrm{Au} 2$} & $6 s$ & 0.353 & 1.039 & 0.363 & 0.910 \\
\hline & $6 p$ & 0.016 & 0.033 & 0.014 & 0.031 \\
\hline & $5 d$ & 9.943 & 9.409 & 9.947 & 9.552 \\
\hline & $4 f$ & 0.002 & 0.002 & 0.002 & 0.002 \\
\hline \multirow[t]{3}{*}{$\mathrm{P}$} & $3 \mathrm{~s}$ & 1.329 & 1.326 & 1.344 & 1.353 \\
\hline & $3 p$ & 3.150 & 3.156 & 3.121 & 3.144 \\
\hline & $3 \mathrm{~d}$ & 0.059 & 0.058 & 0.054 & 0.054 \\
\hline \multirow[t]{3}{*}{$S$} & $3 s$ & 1.666 & 1.673 & 1.668 & 1.668 \\
\hline & $3 p$ & 4.249 & 4.164 & 4.262 & 4.211 \\
\hline & $3 d$ & 0.038 & 0.039 & 0.036 & 0.035 \\
\hline \multirow[t]{2}{*}{ N1 } & $2 s$ & & & 1.573 & 1.562 \\
\hline & $2 p$ & & & 4.044 & 4.036 \\
\hline \multirow[t]{2}{*}{$\mathrm{N} 2$} & $2 \mathrm{~s}$ & & & 1.559 & 1.559 \\
\hline & $2 p$ & & & 4.038 & 4.035 \\
\hline \multicolumn{2}{|c|}{ Wavelength (nm) } & \multicolumn{2}{|c|}{383} & \multicolumn{2}{|c|}{473} \\
\hline
\end{tabular}

S-Table 2. The system energy of the ground state for head-to-tail $\left[\mathrm{Au}\left(\mathrm{PH}_{2} \mathrm{CH}_{2} \mathrm{SH}\right)\right]_{2}{ }^{2+}(1)$, headto-head $\left[\mathrm{Au}\left(\mathrm{PH}_{2} \mathrm{CH}_{2} \mathrm{SH}\right)\right]_{2}{ }^{2+}(3)$, head-to-tail $\left[\mathrm{Au}\left(\mathrm{PH}_{2} \mathrm{CH}_{2} \mathrm{SH}\right)\right]_{2}{ }^{2+} \cdot(\mathrm{MeCN})_{2}(2)$ and head-to-head $\left[\mathrm{Au}\left(\mathrm{PH}_{2} \mathrm{CH}_{2} \mathrm{SH}\right)\right]_{2}{ }^{2+} \cdot(\mathrm{MeCN})_{2}(4)$ under the MP2 calculations.

\begin{tabular}{|c|c|c|c|c|}
\hline & 1 & 3 & 2 & 4 \\
\hline \multirow{2}{*}{ MP2 (Hartree) } & -383.5533164 & -383.5497247 & -647.9847311 & -647.9640510 \\
\hline & Stable* & & stable & \\
\hline
\end{tabular}

* The real complex $\left[\mathrm{Au}_{2}\left(\mathrm{PPh}_{2} \mathrm{CH}_{2} \mathrm{SPh}\right)_{2}\right]\left(\mathrm{CF}_{3} \mathrm{SO}_{3}\right)_{2}$ corresponding to 1 has been synthesized. 


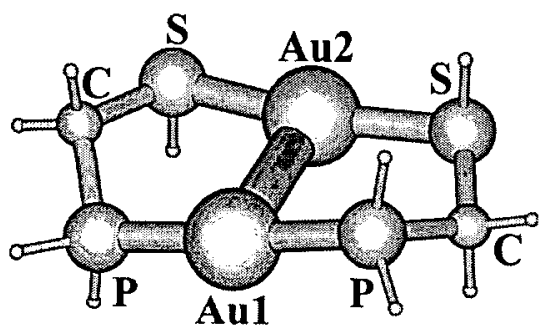

3a

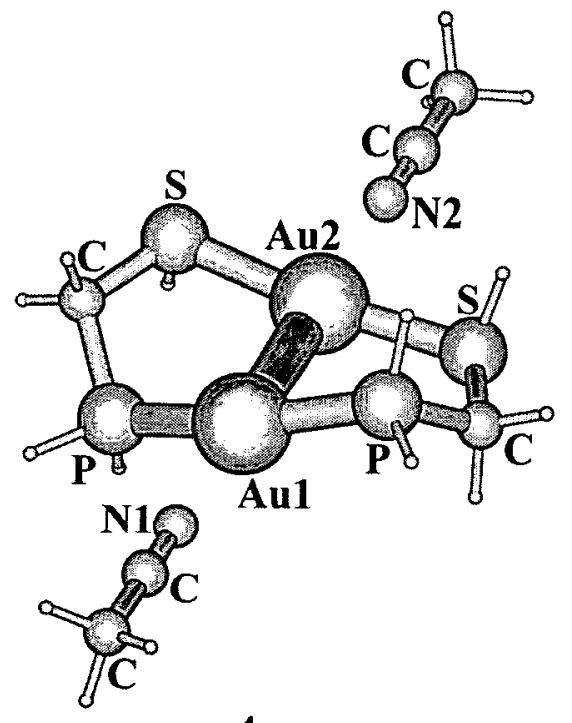

$4 a$

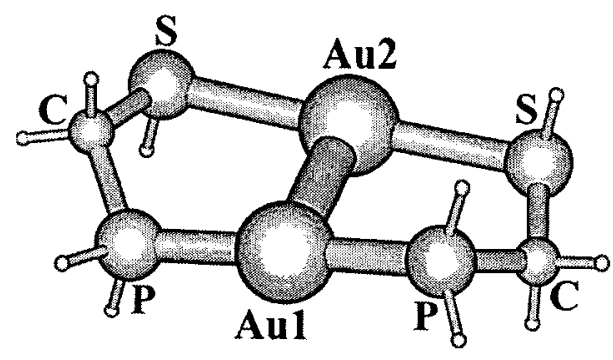

3b

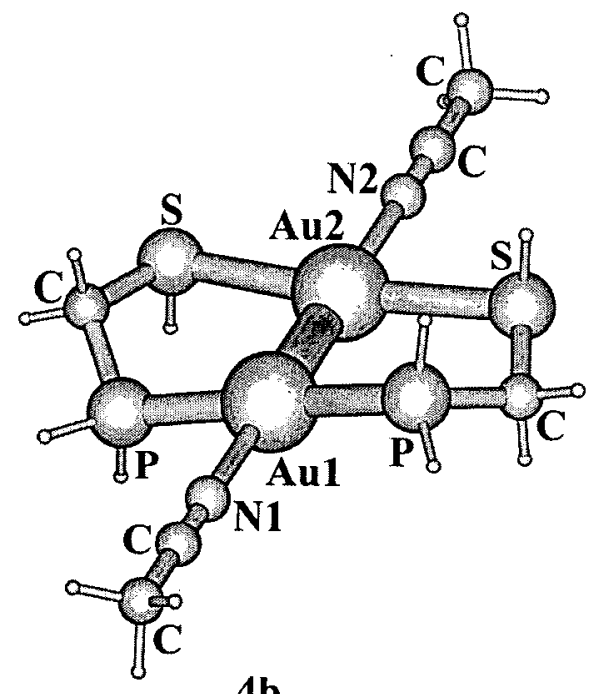

4b

S-Figure I. The optimized ground- and excited-state structures by the MP2 and CIS methods respectively for head-to-head $\left[\mathrm{Au}_{2}\left(\mathrm{PH}_{2} \mathrm{CH}_{2} \mathrm{SH}\right)_{2}\right]^{2+} \quad(3 \mathbf{a} / 3 \mathbf{b})$ and head-to-head $\left[\mathrm{Au}_{2}\left(\mathrm{PH}_{2} \mathrm{CH}_{2} \mathrm{SH}\right)_{2}\right]^{2+} \cdot(\mathrm{MeCN})_{2}(\mathbf{4 a} / \mathbf{4} \mathbf{b})$.

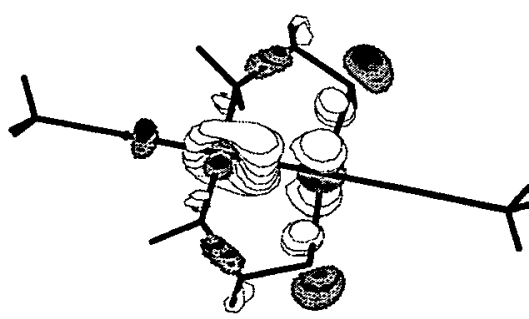

HOMO-2
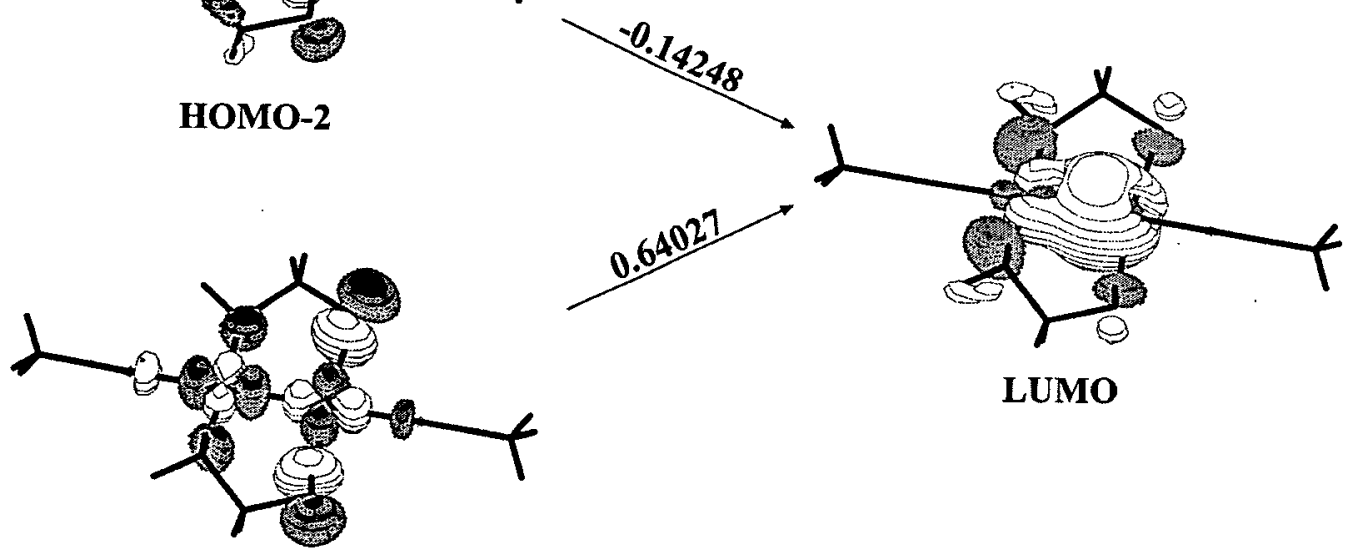

HOMO

S-Figure II. The single electron transitions with $\mid \mathrm{CI}$ coefficient $\mid>0.1$ in the CIS calculation for $473 \mathrm{~nm}$ phosphorescence of head-to-head $\left[\mathrm{Au}_{2}\left(\mathrm{PH}_{2} \mathrm{CH}_{2} \mathrm{SH}\right)_{2}\right]^{2+} \cdot(\mathrm{MeCN})_{2}(4)$. 
S-Table 3. Phosphorescence wavelengths and the natural atomic orbital populations of the ${ }^{3} \mathrm{~A}_{u}$ excited state and the corresponding ${ }^{1} \mathrm{~A}_{\mathrm{g}}$ ground state for head-to-tail $\left[\mathrm{Au}_{2}\left(\mathrm{PH}_{2} \mathrm{CH}_{2} \mathrm{SCH}_{3}\right)_{2}\right]^{2+}(5)$ and head-to-tail $\left[\mathrm{Au}_{2}\left(\mathrm{PH}_{2} \mathrm{CH}_{2} \mathrm{SCH}_{3}\right)_{2}\right]_{2}{ }^{2+} \cdot(\mathrm{MeCN})_{2}(6)$ under the CIS calculations.

\begin{tabular}{|c|c|c|c|c|c|}
\hline \multirow{2}{*}{ Atoms } & \multirow{2}{*}{ Orbitals } & \multicolumn{2}{|c|}{5} & \multicolumn{2}{|c|}{6} \\
\hline & & ${ }^{1} \mathrm{~A}_{\mathrm{g}}$ & ${ }^{3} \mathrm{~A}_{\mathrm{u}}$ & ${ }^{1} \mathrm{~A}_{\mathrm{g}}$ & ${ }^{3} \mathrm{~A}_{\mathrm{u}}$ \\
\hline \multirow[t]{4}{*}{$\mathrm{Au}$} & $6 s$ & 0.625 & 0.779 & 0.483 & 0.773 \\
\hline & $6 p$ & 0.017 & 0.083 & 0.017 & 0.036 \\
\hline & $5 d$ & 9.869 & 9.634 & 9.893 & 9.624 \\
\hline & $4 \mathrm{f}$ & 0.003 & 0.004 & 0.002 & 0.003 \\
\hline \multirow[t]{3}{*}{$\mathbf{P}$} & $3 \mathrm{~s}$ & 1.346 & 1.352 & 1.362 & 1.360 \\
\hline & $3 p$ & 3.140 & 3.155 & 3.140 & 3.116 \\
\hline & $3 d$ & 0.056 & 0.055 & 0.052 & 0.051 \\
\hline \multirow[t]{3}{*}{$S$} & $3 s$ & 1.612 & 1.617 & 1.624 & 1.627 \\
\hline & $3 p$ & 4.115 & 4.081 & 4.128 & 4.116 \\
\hline & $3 d$ & 0.037 & 0.036 & 0.032 & 0.032 \\
\hline \multirow[t]{2}{*}{$\mathrm{Cl}$} & $2 \mathrm{~s}$ & 1.205 & 1.211 & 1.201 & 1.203 \\
\hline & $2 p$ & 3.620 & 3.621 & 3.625 & 3.624 \\
\hline $\mathrm{H}_{\mathrm{Cl}}$ & Is & 0.726 & 0.727 & 0.735 & 0.738 \\
\hline $\mathrm{H}_{\mathrm{Cl}}$ & $1 \mathrm{~s}$ & 0.722 & 0.722 & 0.731 & 0.732 \\
\hline $\mathrm{H}_{\mathrm{Pl}}$ & $1 \mathrm{~s}$ & 0.993 & 0.995 & 1.015 & 1.017 \\
\hline $\mathrm{H}_{\mathrm{Pl}}$ & $1 \mathrm{~s}$ & 0.965 & 0.974 & 0.994 & 1.001 \\
\hline \multirow[t]{2}{*}{$\mathrm{C}_{\mathrm{S}}$} & $2 \mathrm{~s}$ & 1.172 & 1.173 & 1.168 & 1.169 \\
\hline & $2 p$ & 3.510 & 3.513 & 3.520 & 3.522 \\
\hline \multirow[t]{2}{*}{$\mathrm{N}$} & $2 s$ & & & 1.565 & 1.555 \\
\hline & $2 p$ & & & 4.052 & 4.045 \\
\hline \multirow[t]{2}{*}{$\mathrm{C} 2$} & $2 s$ & & & 0.887 & 0.886 \\
\hline & $2 p$ & & & 2.582 & 2.583 \\
\hline \multirow[t]{2}{*}{$\mathrm{C} 3$} & $2 s$ & & & 1.124 & 1.124 \\
\hline & $2 p$ & & & 3.552 & 3.552 \\
\hline $\mathrm{H}_{\mathrm{C} 3}$ & $1 \mathrm{~s}$ & & & 0.735 & 0.735 \\
\hline \multicolumn{2}{|c|}{ Wavelength (nm) } & \multicolumn{2}{|c|}{332} & \multicolumn{2}{|c|}{585} \\
\hline
\end{tabular}


(C) $200 \overline{3}$ American Chemical Society, Inorg. Chem., Pan ic0300159 Supporting Info Page 4

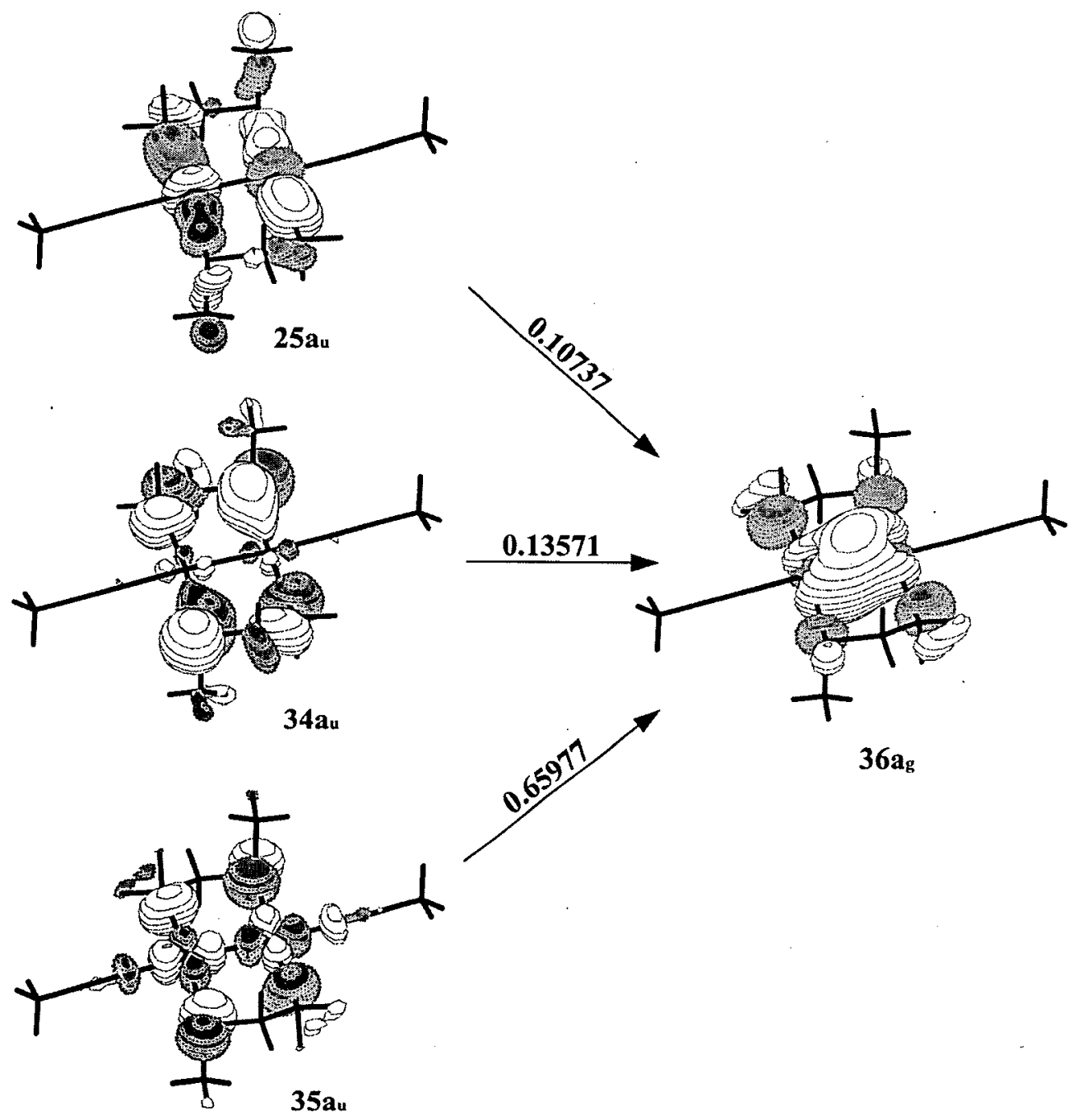

S-Figure III. The single electron transitions with $\mid \mathrm{CI}$ coefficient $\mid>0.1$ in the CIS calculation for $585 \mathrm{~nm}$ phosphorescence of $\left[\mathrm{Au}_{2}\left(\mathrm{PH}_{2} \mathrm{CH}_{2} \mathrm{SCH}_{3}\right)_{2}\right]^{2+} \cdot(\mathrm{MeCN})_{2}(6)$

S-Table 4. The optimized main geometry parameters of the ${ }^{1} \mathrm{~A}_{\mathrm{g}}$ ground state and the ${ }^{3} \mathrm{~A}_{\mathrm{u}}$ excited state for head-to-tail $\left[\mathrm{Au}_{2}\left(\mathrm{PH}_{2} \mathrm{CH}_{2} \mathrm{~S}\right)_{2}\right]$ (7) using the MP2 and CIS methods respectively.*

\begin{tabular}{ccc}
\hline Parameters & ${ }^{1} \mathrm{~A}_{\mathrm{g}}$ & ${ }^{3} \mathrm{~A}_{\mathrm{u}}$ \\
\hline Bond length $(\AA)$ & & \\
$\mathrm{Au}-\mathrm{Au}$ & 2.988 & 2.728 \\
$\mathrm{Au}-\mathrm{P}$ & 2.331 & 2.524 \\
$\mathrm{Au}-\mathrm{S}$ & 2.358 & 2.463 \\
P...S & 3.047 & 3.103 \\
Bond angle $\left(^{\circ}\right)$ & & \\
P-Au-S & 178.4 & 171.3 \\
P-Au-Au & 82.8 & 91.0 \\
S-Au-Au & 98.6 & 97.7 \\
Dihedral angle $\left(^{\circ}\right)$ & & 179.3 \\
P-Au-Au-S & 179.2 & 125.0 \\
C-P-S-Au & 122.8 & \\
\hline Wavelength $(\mathrm{nm})$ & & 323 \\
\hline
\end{tabular}

* The calculated results from ref. 36 . 
S-Table 5. The natural atomic orbital populations of the ${ }^{3} \mathrm{~A}_{\mathrm{u}}$ excited state and the corresponding ${ }^{1} \mathrm{~A}_{\mathrm{g}}$ ground state for $\left[\mathrm{Au}_{2}\left(\mathrm{PH}_{2} \mathrm{CH}_{2} \mathrm{~S}\right)_{2}\right](7)$ involved in the $323 \mathrm{~nm}$ emission.*

\begin{tabular}{cccc}
\hline Atoms & Orbitals & ${ }^{1} \mathrm{~A}_{\mathrm{g}}$ & ${ }^{3} \mathrm{~A}_{\mathrm{u}}$ \\
\hline $\mathrm{Au}$ & $6 \mathrm{~s}$ & 0.735 & 0.802 \\
& $6 \mathrm{p}$ & 0.024 & 0.138 \\
& $5 \mathrm{~d}$ & 9.839 & 9.687 \\
$\mathrm{P}$ & $4 \mathrm{f}$ & 0.003 & 0.005 \\
& $3 \mathrm{~s}$ & 1.358 & 1.366 \\
& $3 \mathrm{p}$ & 3.133 & 3.209 \\
$\mathrm{~S}$ & $3 \mathrm{~d}$ & 0.053 & 0.053 \\
& $3 \mathrm{~s}$ & 1.762 & 1.765 \\
& $3 \mathrm{p}$ & 4.665 & 4.522 \\
$\mathrm{C}$ & $3 \mathrm{~d}$ & 0.023 & 0.023 \\
& $2 \mathrm{~s}$ & 1.192 & 1.206 \\
$\mathrm{H}_{\mathrm{C}}$ & $2 \mathrm{p}$ & 3.612 & 3.616 \\
$\mathrm{H}_{\mathrm{C}}$ & $1 \mathrm{~s}$ & 0.769 & 0.766 \\
$\mathrm{H}_{\mathrm{P}}$ & $1 \mathrm{~s}$ & 0.768 & 0.768 \\
$\mathrm{H}_{\mathrm{P}}$ & $1 \mathrm{~s}$ & 1.026 & 1.025 \\
\hline
\end{tabular}

* The natural atomic orbital population was listed in the table, but the Mulliken population was employed in ref. 36.

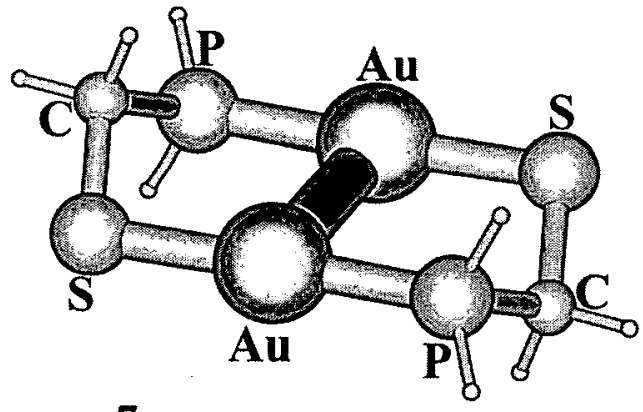

$7 \mathbf{a}$

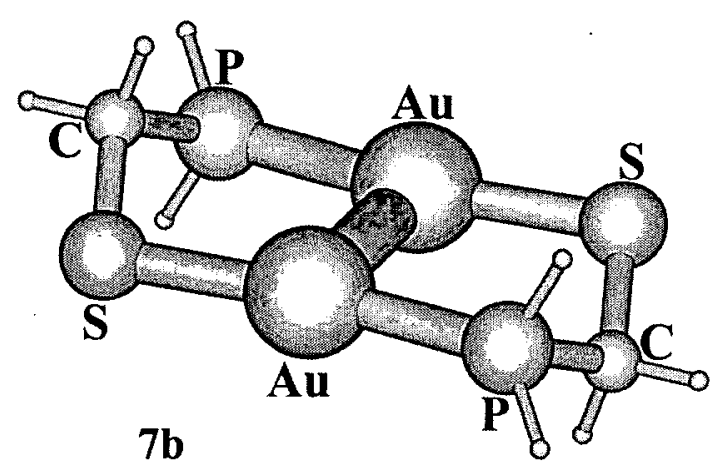

$7 \mathbf{b}$

S-Figure IV. The optimized ${ }^{1} \mathrm{~A}_{\mathrm{g}}$ ground-state structure and ${ }^{3} \mathrm{~A}_{\mathrm{u}}$ excited-state structure for head-totail $\left[\mathrm{Au}_{2}\left(\mathrm{PH}_{2} \mathrm{CH}_{2} \mathrm{~S}\right)_{2}\right]$ (7) using the MP2 and CIS methods respectively. 
(C) 2003 American Chemical Society, Inorg. Chem., Pan ic0300159 Supporting Info Page 6

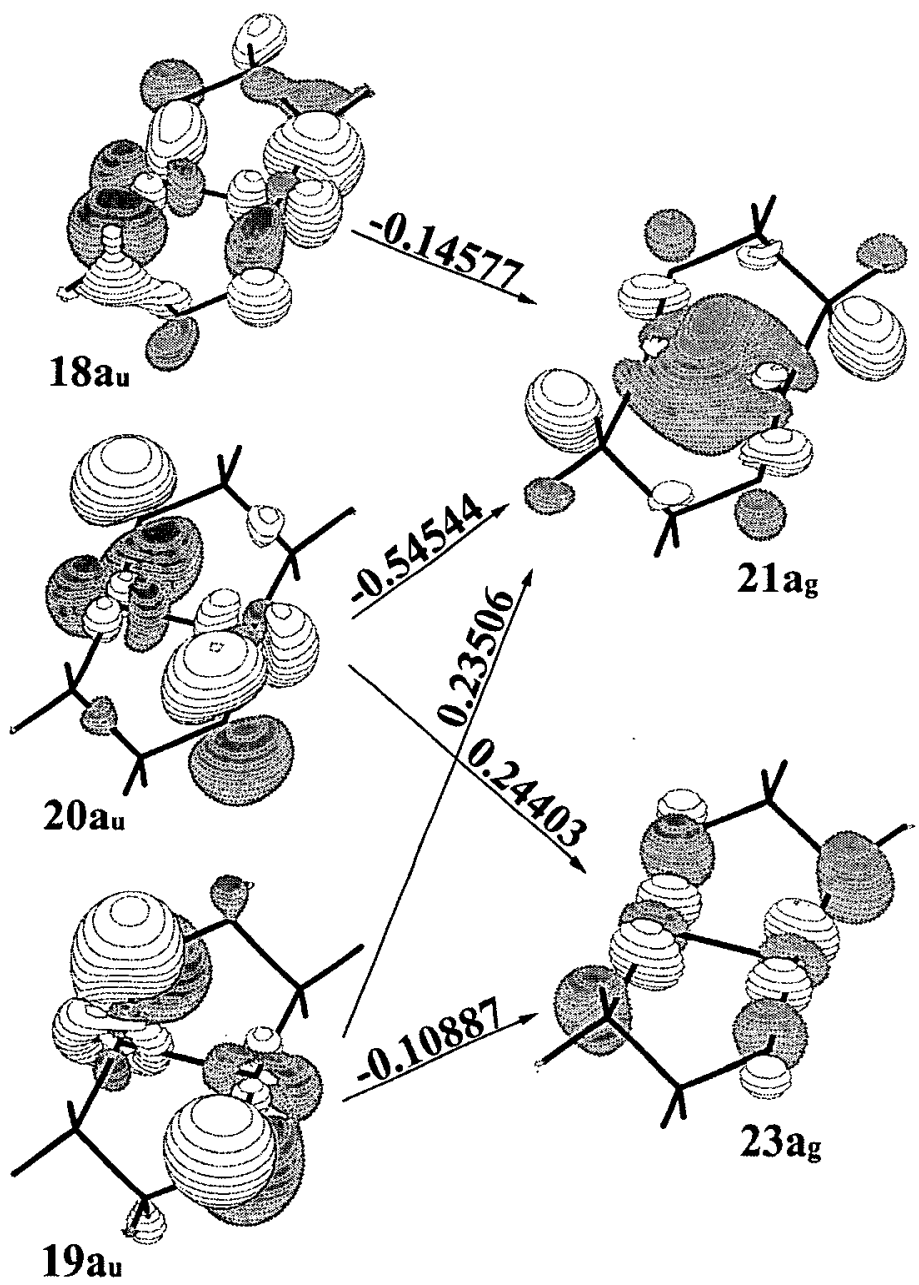

S-Figure $V$. The single electron transition with $\mid C I$ coefficient $\mid>0.1$ in the CIS calculation for the $323 \mathrm{~nm}$ triplet emission of $\left[\mathrm{Au}_{2}\left(\mathrm{PH}_{2} \mathrm{CH}_{2} \mathrm{~S}\right)\right](7)$.

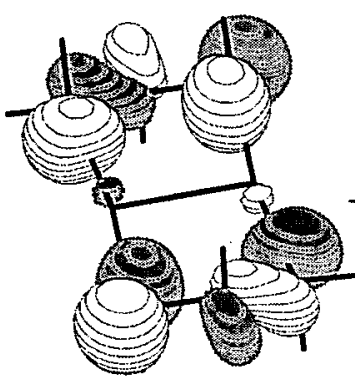

$19 a_{u}$

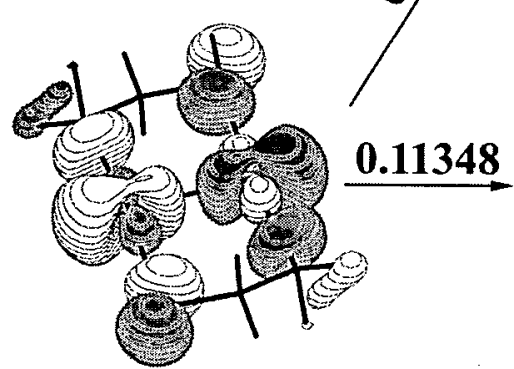

$20 a_{u}$

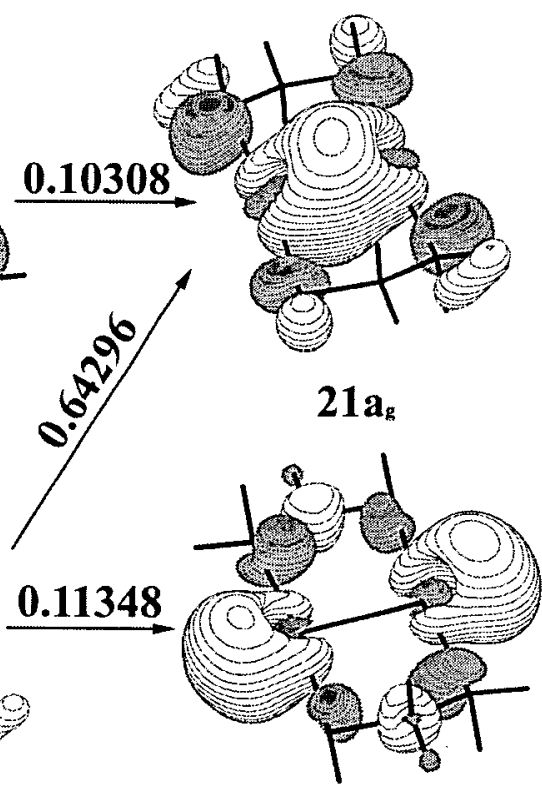

$22 a_{k}$

S-Figure VI. The single electron transitions with $\mid \mathrm{CI}$ coefficient $\mid>0.1$ in the CIS calculation for $337 \mathrm{~nm}$ phosphorescence of $\left[\mathrm{Au}_{2}\left(\mathrm{PH}_{2} \mathrm{CH}_{2} \mathrm{SH}\right)_{2}\right]^{2+}(1)$. 